\title{
U-shaped strap muscle flap for difficult thyroid surgery
}

\author{
How-Yun Ko ${ }^{1}$, I-Cheng Lu ${ }^{2,3}$, Pi-Ying Chang ${ }^{3}$, Ling-Feng Wang ${ }^{1,2}$, Che-Wei Wu ${ }^{1,2}$, Wing-Hei Viola Yu ${ }^{1}$, \\ Tzer Zen Hwang ${ }^{4,5}$, Chien Chung Wang ${ }^{5}$, Tzu-Yen Huang ${ }^{1,6}$, Feng-Yu Chiang ${ }^{4,5}$
}

${ }^{1}$ Department of Otolaryngology-Head and Neck Surgery, Kaohsiung Medical University Hospital, Kaohsiung Medical University, Kaohsiung, Taiwan; ${ }^{2}$ Faculty of Medicine, College of Medicine, Kaohsiung Medical University, Kaohsiung, Taiwan; ${ }^{3}$ Department of Anesthesiology, Kaohsiung Medical University Hospital, Kaohsiung Medical University, Kaohsiung, Taiwan; ${ }^{4}$ School of Medicine, College of Medicine, I-Shou University, Kaohsiung, Taiwan; ${ }^{5}$ Department of Otolaryngology, E-Da Hospital, Kaohsiung, Taiwan; ${ }^{6}$ Department of Biological Science and Technology, National Chiao Tung University, Hsinchu, Taiwan

Contributions: (I) Conception and design: FY Chiang, TY Huang; (II) Administrative support: FY Chiang, TY Huang; (III) Provision of study materials or patients: FY Chiang; (IV) Collection and assembly of data: HY Ko ,TY Huang; (V) Data analysis and interpretation: HY Ko ,TY Huang; (VI) Manuscript writing: All authors; (VII) Final approval of manuscript: All authors.

Correspondence to: Feng-Yu Chiang, MD. Department of Otolaryngology, E-Da Hospital, I-Shou University, No.1, Yida Road, Yanchao District, Kaohsiung 82445, Taiwan. Email: fychiang@kmu.edu.tw; Tzu-Yen Huang, MD. Department of Otolaryngology-Head and Neck Surgery, Kaohsiung Medical University Hospital, 100 Tzyou First Road, Kaohsiung 807, Taiwan. Email: tyhuang.ent@gmail.com.

Backgroundz Open thyroidectomy via conventional midline approach can be challenging in complex
thyroid surgeries. This study proposes a U-shaped strap muscle flap (USMF) technique that provides
adequately wide exposure of the surgical field.
Methods: Strap muscles were cut close to the clavicle and along the anterior margin of both
sternocleidomastoid muscles followed by total thyroidectomy in 20 patients as USMF group, and surgical
outcomes were compared with 40 patients who had received total thyroidectomy via midline approach.
Results: No patient had postoperative hematoma, vocal cord paralysis, permanent hypocalcaemia, wound
infection or flap necrosis. At 2 months post-surgery, objective voice analysis and subjective assessment of
voice and swallowing showed no significant difference between groups.

Conclusions: USMF provides superb surgical field exposure, and the voice and swallowing functions after USMF are comparable to those obtained by midline approach. The USMF approach is a feasible option for selective difficult thyroid surgery.

Keywords: U-shaped strap muscle flap (USMF); thyroid; voice; swallowing; thyroidectomy

Submitted Feb 01, 2020. Accepted for publication Feb 14, 2020.

doi: 10.21037 /gs.2020.02.20

View this article at: http://dx.doi.org/10.21037/gs.2020.02.20

\section{Introduction}

Wide and adequate exposure of the surgical field is essential for successful thyroid surgery. The most common procedure for conventional open thyroidectomy is the midline approach, in which the strap muscles are divided along the linea alba and separated from the thyroid gland. However, midline approach may be difficult to perform in some patients, including patients with huge goiter, large Graves' disease, thyroid cancer with lateral neck metastasis, obesity and short neck. An insufficient surgical field exposure not only increases operation time and blood loss, it also increases the risk of injury to the recurrent laryngeal nerve (RLN) and parathyroid gland (PG) due to poor visibility during surgical dissection (1).

The aim of this study was to evaluate the U-shaped strap muscle flap (USMF) approach that provides excellent exposure of the surgical field. Postoperative objective voice analysis and subjective assessment of voice and swallowing were also performed to evaluate USMF outcomes, including voice and swallowing function. 

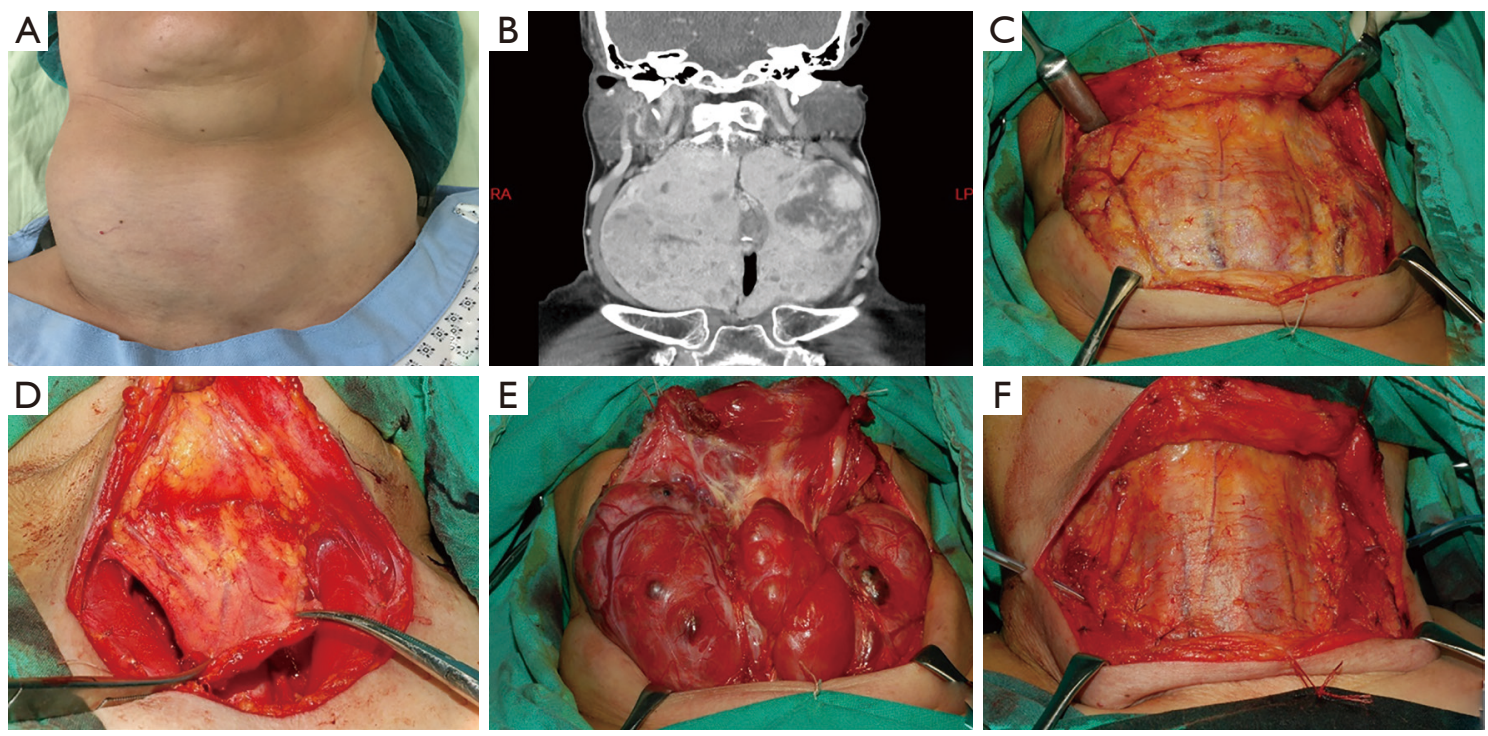

Figure 1 Thyroid surgeries via U-shaped strap muscle flap (USMF) approach. Huge goiter $(16 \mathrm{~cm} \times 13 \mathrm{~cm})$ with tracheal compression: (A) appearance; (B) computed tomography image. (C) conventional open thyroidectomy with adequate skin flap elevation; (D) straps muscles were cut at lower margin close to the clavicle and cut along the anterior margin of both SCM muscle; (E) the strap muscles were separated from the thyroid gland and elevated to the superior level of thyroid cartilage to achieve wide and adequate exposure of surgical field for total thyroidectomy; (F) USMF was sutured back to the original position. SCM, sternocleidomastoid muscle.

\section{Methods}

From January, 2017 to June, 2018, this study enrolled 20 patients who had received total thyroidectomy by USMF approach, including 15 with huge goiters, 2 with large Graves' disease, and 3 with thyroid cancers with lateral neck metastasis. The USMF was performed as follows. After elevation of the skin flap, the strap muscles were cut at a lower margin close to the clavicle and then cut along the anterior margin of both sternocleidomastoid muscles. The strap muscles were then separated from the thyroid gland and elevated to the superior level of thyroid cartilage (Video 1). After complete resection of tumors, the muscle flap was sutured back to its original position. Figure 1 shows the USMF approach for a huge goiter, and Figure 2 shows the USMF approach for thyroid cancer with lateral neck metastasis.

In all patients, analyses of ionized calcium (iCa) level and vocal cord function, objective analysis of voice, and subjective analyses of voice and swallowing were performed before surgery and 2 months after surgery. Another 40 patients who had received total thyroidectomy by midline approach during the same time period were used as a control group to compare voice and swallowing outcomes. All surgeries were performed under neural monitoring by a single surgeon (FY Chiang).

\section{Evaluation of serum iCa and vocal cord function}

In all patients, $\mathrm{iCa}$ was measured before and $12,24,48$, and 72 hours after surgery. Postoperative hypocalcemia $(\mathrm{PH})$ was considered permanent if iCa did not recover and calcitriol/ calcium supplements could not be withdrawn 12 months after surgery. Vocal cord mobility was routinely video-recorded by flexible laryngofiberscopy before and after surgery. A dysfunction was considered permanent if abnormal vocal cord movement persisted 6 months after surgery.

\section{Objective voice analysis}

Objective voice analysis was performed preoperatively and 2 months postoperatively. The assessments were performed by an experienced speech-language pathologist using Multidimensional Voice Program (MDVP, model 5105, version 3.1.7; KayPENTAX, USA) and Voice Range Profile (VRP, model 4326, version 3.3.0; KayPENTAX, USA). Maximum pitch frequency (Fmax), minimum pitch frequency (Fmin), pitch range (PR), maximum phonation time (MPT), 

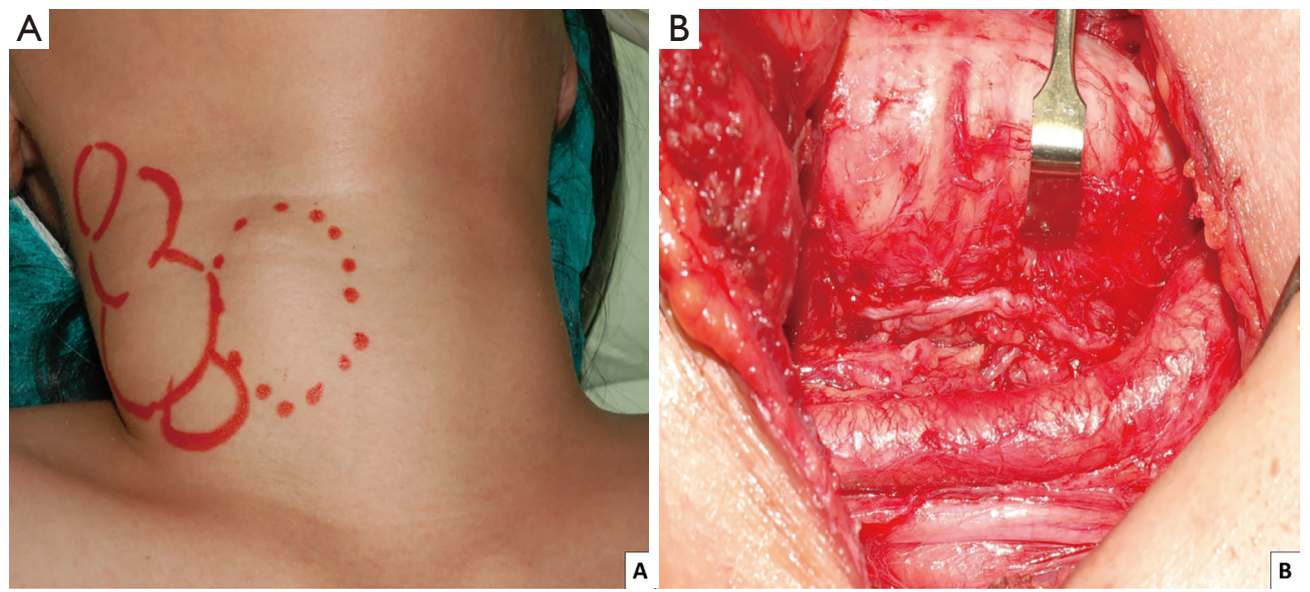

Figure 2 Thyroid cancer with lateral neck metastasis (A) and outcome of USMF approach in a total thyroidectomy with central and lateral neck dissection (B).

Table 1 Subjective voice and swallowing evaluation questionnaires

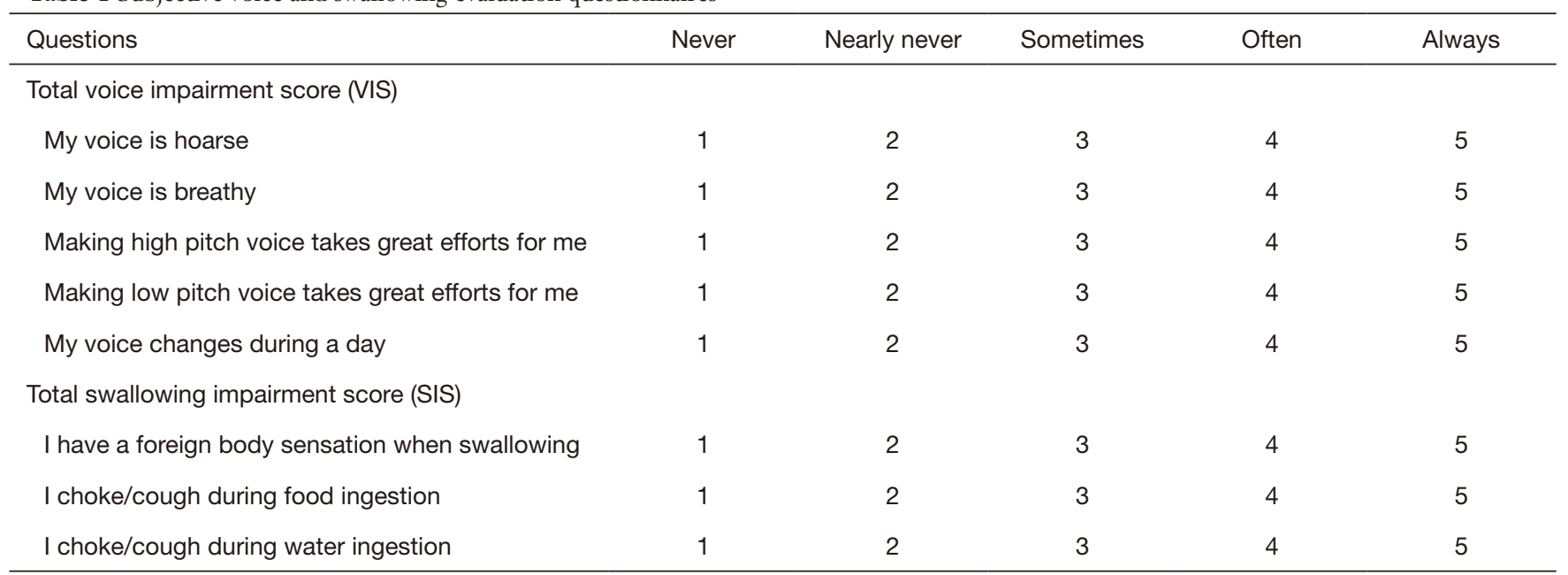

mean fundamental frequency (mean F0), Jitter (Jitt), and Shimmer (Shim) were recorded. The PR was defined as the number of semitones between Fmax and Fmin.

\section{Subjective assessment of voice and swallowing}

The severity of voice and swallowing abnormalities was evaluated by questionnaires (Table 1). The scores resulting from the questionnaires were defined as voice impairment score (VIS) and swallowing impairment score (SIS). The VIS and SIS scores ranged from 1 (no impairment of voice or swallowing) to 5 (severe impairment of voice or swallowing).

\section{Calculation of postoperative change in voice analysis data}

The equation used to calculate postoperative changes in voice analysis data was

$$
\Delta=100 \% \text { * (B - A }) / A
$$

Where $\mathrm{A}$ and $\mathrm{B}$ are preoperative and postoperative values, respectively.

\section{Statistical analysis}

The statistical software used for all t-tests was SPSS (Version 18.0 for windows; SPSS Inc., Chicago, IL, USA). A P value less than 0.05 was considered statistically significant. This study was approved by the Kaohsiung Medical University 
Table 2 Demographic and clinical characteristics of USMF group and midline group

\begin{tabular}{|c|c|c|c|}
\hline Variables & USMF group (20 patients) & Midline group (40 patients) & $P$ value \\
\hline Age (SD) (years) & $50.52(12.36)$ & $52.68(12.12)$ & 0.434 \\
\hline Histopathology (benign/malignant) & $15 / 5$ & $32 / 8$ & 0.674 \\
\hline \multicolumn{4}{|l|}{$\mathrm{PH}$} \\
\hline Permanent PH (\%) & $0(0.0)$ & $0(0.0)$ & 1.000 \\
\hline \multicolumn{4}{|l|}{ Vocal cord palsy ( $\%$ of nerves at risk) } \\
\hline Temporary palsy & $0(0.0)$ & $0(0.0)$ & 1.000 \\
\hline Permanent palsy & $0(0.0)$ & $0(0.0)$ & 1.000 \\
\hline Fmax & $666.13 \pm 163.62$ & $712.83 \pm 208.35$ & 0.498 \\
\hline Fmin & $119.65 \pm 25.74$ & $121.88 \pm 33.62$ & 0.914 \\
\hline Pitch range & $28.18 \pm 4.86$ & $30.08 \pm 6.78$ & 0.114 \\
\hline MPT & $18.91 \pm 5.23$ & $20.85 \pm 8.04$ & 0.151 \\
\hline Mean F0 & $204.64 \pm 42.59$ & $204.64 \pm 42.59$ & 0.183 \\
\hline Jitter & $0.86 \pm 0.44$ & $0.81 \pm 0.42$ & 0.336 \\
\hline Shimmer & $2.27 \pm 0.88$ & $2.22 \pm 0.88$ & 0.143 \\
\hline VIS & $16.13 \pm 6.87$ & $9.45 \pm 6.51$ & 0.001 \\
\hline
\end{tabular}

USMF, U-shaped strap muscle flap; SD, standard deviation; PH, postoperative hypocalcemia; Fmax, maximum pitch frequency; Fmin, minimum pitch frequency; MPT, maximum phonation time; mean F0, mean fundamental frequency; VIS, voice impairment score; SIS, swallowing impairment score.

Hospital Institutional Review Board [No. KMUH-IRB$\mathrm{E}(\mathrm{I})-20190201]$.

\section{Results}

All 20 thyroid surgeries performed by USMF approach were uneventful and had no adverse events (e.g., no postoperative hematoma, wound infection or flap necrosis).

The USMF group and the midline group did not significantly differ in gender, mean age, histopathology, $\mathrm{PH}$ rate or vocal cord palsy. Temporary $\mathrm{PH}$ occurred in $3(15.0 \%)$ patients in the USMF group and in $2(5.0 \%)$ patients in the midline group, and no patients had permanent PH. Neither group had patients with transient or permanent vocal cord palsy. Preoperative objective voice analysis revealed that no parameters differed between the USMF group and the midline group. Between-group comparisons revealed that the USMF group had significantly higher VIS $(16.13 \pm 6.87$ vs. $9.45 \pm 6.51$ in midline group, $\mathrm{P}=0.001)$ and significantly higher SIS $(9.70 \pm 3.91$ vs. $6.25 \pm 4.32$ in midline group, $\mathrm{P}=0.001)$. Table 2 shows the results of preoperative subjective assessments of voice and swallowing.

Table 3 compares preoperative and 2 -month postoperative voice and swallowing outcomes. Objective voice analysis at 2 months post-surgery showed significantly lower Fmax and PR in both groups. Subjective assessments revealed no significant VIS change in the USMF group (preoperative VIS $16.13 \pm 6.87 v s$. postoperative VIS $15.70 \pm 4.91 ; \mathrm{P}=0.010$ ). In the midline group, however, VIS significantly increased (preoperative VIS $9.45 \pm 6.51$ vs. postoperative VIS $10.25 \pm 7.28 ; \mathrm{P}=0.010)$. In contrast, SIS did not significantly differ in either the USMF group (preoperative SIS 9.70 \pm 3.91 
Table 3 Comparison of preoperative and postoperative 2 months voice and swallowing outcomes in USMF and midline group

\begin{tabular}{|c|c|c|c|c|c|c|}
\hline Variables & $\begin{array}{l}\text { USMF preoperative, } \\
\text { mean }( \pm S D)\end{array}$ & $\begin{array}{c}\text { USMF } 2 \text { months } \\
\text { postoperative, mean }( \pm S D)\end{array}$ & $P$ value & $\begin{array}{l}\text { Midline preoperative, } \\
\text { mean }( \pm S D)\end{array}$ & $\begin{array}{c}\text { Midline, } 2 \text { months } \\
\text { postoperative, mean }( \pm S D)\end{array}$ & $P$ value \\
\hline $\mathrm{Fmax}(\mathrm{Hz})$ & $666.13 \pm 163.62$ & $592.70 \pm 202.19$ & 0.001 & $712.83 \pm 208.35$ & $638.90 \pm 248.47$ & 0.017 \\
\hline Pitch range & $28.18 \pm 4.86$ & $25.20 \pm 7.02$ & 0.003 & $30.08 \pm 6.78$ & $26.88 \pm 8.00$ & 0.004 \\
\hline Jitter (\%) & $0.86 \pm 0.44$ & $0.98 \pm 0.52$ & ns & $0.81 \pm 0.42$ & $0.95 \pm 0.62$ & ns \\
\hline Shimmer (\%) & $2.27 \pm 0.88$ & $2.71 \pm 1.31$ & ns & $2.22 \pm 0.88$ & $2.53 \pm 1.25$ & ns \\
\hline VIS & $16.13 \pm 6.87$ & $15.70 \pm 4.91$ & ns & $9.45 \pm 6.51$ & $10.25 \pm 7.28$ & 0.010 \\
\hline
\end{tabular}

USMF, U-shaped strap muscle flap; SD, standard deviation; Fmax, maximum pitch frequency; Fmin, minimum pitch frequency; MPT, maximum phonation time; mean F0, mean fundamental frequency; VIS, voice impairment score; SIS, swallowing impairment score; ns, not significant.

Table 4 Comparison of the difference of preoperative and postoperative 2 months objective voice analysis and subjective assessment of voice and swallowing in two groups

\begin{tabular}{lccc}
\hline Variables & USMF group & Midline group & P value \\
\hline$\Delta$ Fmax & $-19.27 \pm 22.66$ & $-9.92 \pm 25.01$ & $\mathrm{~ns}$ \\
$\Delta$ Fmin & $17.84 \pm 47.51$ & $7.73 \pm 25.62$ & $\mathrm{~ns}$ \\
$\Delta$ Pitch range & $-17.41 \pm 22.16$ & $-9.69 \pm 19.95$ & $\mathrm{~ns}$ \\
$\Delta$ MPT & $8.32 \pm 35.46$ & $3.02 \pm 38.00$ & $\mathrm{~ns}$ \\
$\Delta$ Mean F0 & $-0.97 \pm 11.17$ & $-3.25 \pm 14.84$ & $\mathrm{~ns}$ \\
$\Delta$ Jitter & $49.52 \pm 171.75$ & $51.44 \pm 118.81$ & $\mathrm{~ns}$ \\
$\Delta$ Shimmer & $22.56 \pm 82.45$ & $25.44 \pm 67.08$ & $\mathrm{~ns}$ \\
$\Delta$ VIS & $-1.05 \pm 30.19$ & $-4.96 \pm 12.24$ & $\mathrm{~ns}$ \\
$\Delta$ SIS & $0.80 \pm 29.92$ & $6.96 \pm 46.06$ & $\mathrm{~ns}$
\end{tabular}

For the calculation formula of $\Delta$, please refer to the method. USMF, U-shaped strap muscle flap; Fmax, maximum pitch frequency; Fmin, minimum pitch frequency; MPT, maximum phonation time; mean F0, mean fundamental frequency; VIS, voice impairment score; SIS, swallowing impairment score; ns, not significant.

vs. postoperative SIS $9.10 \pm 2.57 ; \mathrm{P}>0.05)$ or in the midline group (preoperative SIS $6.25 \pm 4.32 v s$. postoperative SIS $6.48 \pm 4.60 ; \mathrm{P}>0.05)$.

Between-group comparisons of preoperative and 2 -month postoperative $\Delta$ value of objective voice analysis and subjective assessment of voice and swallowing showed no significant difference (Table 4).

\section{Discussion}

Large goiter, Graves' disease and thyroid cancer are associated with increased complication rates in thyroid surgery. For example, after thyroidectomy, large thyroid glands and Graves' disease are risk factors for hematoma and prolonged hypocalcemia (2-4). Thyroid cancer patients who have received neck dissection also have an increased risk of hypoparathyroidism and RLN injury after thyroidectomy $(5,6)$. The major complications of thyroid surgery include RLN injury, external branch of superior laryngeal nerve (EBSLN) injury, and permanent hypoparathyroidism, besides, over-traction of tissue or prolonged surgical time also results in postoperative sequelae (7). Therefore, 
adequate exposure of the surgical field and meticulous dissection are essential for a successful thyroid surgery. The USMF approach is a selectable method to minimize these complications by providing wide surgical exposure.

The reported effects of partial or complete resection of strap muscles on voice and swallowing performance widely vary. A comprehensive literature review by Vilkman et al. (8) concluded that changes in a complex interactive system such as the external laryngeal frame (including the sternothyroid and sternohyoid muscles) can result in increased or decreased voice pitch. In Sonninen et al. (9), 50 thyroidectomy patients who had received bilateral division of the strap muscles were compared with 50 thyroidectomy patients who had received unilateral division or no division of the strap muscles. The authors concluded that the unilateral group had better high voice pitch outcomes.

However, other studies of unilateral and bilateral strap muscle division $(10,11)$ and sternothyroid muscle division (12) have concluded that these procedures have no subjective or objective functional sequelae. Partial cutting of the sternothyroid muscle at the laryngeal head at the insertion point of the sternothyroid muscle along the thyroid cartilage is reportedly a safe surgical technique for exposing the superior pole of the thyroid gland to facilitate isolation and ligation of the vascular pedicles of the superior pole and for enabling easy identification and preservation of the EBSLN (13).

The reported prevalence of symptoms in preoperative thyroidectomy patients widely varies from $11 \%$ to $88 \%$ (14-16). As thyroid volume increases, preoperative compressive symptoms may increase (14) whereas PR may decrease (17). The preoperative assessments in our study showed that the USMF group had significantly higher VIS and SIS compared with the midline group, which suggests that patients treated by USMF approach may tend to have a larger thyroid volume or greater disease severity compared to those treated by midline approach.

Some studies have reported substantial changes in objective voice analysis results after thyroid surgery, including decreased habitual pitch, decreased vocal performance (e.g., decreased frequency and intensity range), and increased perturbation parameters (e.g., increased shim, jitt, noise-to-harmonic ratio, and voice turbulence index) (18-21). Changes in voice and swallowing have been reported in patients who have received the conventional midline approach $(22,23)$. In our study, Fmax and PR significantly decreased 2 months after both USMF and midline approach. Other parameters did not significantly differ. Lee et al. (13) similarly reported that patients who had received thyroidectomies that required partial cutting of the sternothyroid muscle had decreased Fmax at 2 weeks post-surgery. Lombardi et al. (23) reported that objective parameters recorded preoperatively and at 3 months postsurgery did not significantly differ. Our study similarly showed that VIS significantly increased in the midline group $(\mathrm{P}=0.010)$. In the USMF group, VIS did not significantly differ; in fact, VIS slightly improved $(16.13 \pm 6.87$ preoperatively and $15.70 \pm 4.91$ postoperatively). We hypothesize that the USMF group had larger improvements in VIS because patients in the USMF group was more likely to have large goiter or thyroid tumor decompression.

For an accurate comparison of changes in voice and swallowing parameters, preoperative and postoperative $\Delta$ values were compared between the two groups. Betweengroup comparisons before surgery and 2 months after surgery showed no significant differences in $\Delta$. Therefore, we conclude that the voice and swallowing outcomes obtained by USMF approach were not inferior to those obtained by midline approach.

Several causes of voice alteration in thyroidectomy patients without EBSLN injury or RLN injury have been postulated, including orotracheal intubation, modification of vascular supply and venous drainage of the larynx, impairment of laryngotracheal movement, injury to the cricothyroid muscle, local pain in the neck, and psychological reaction to postoperative outcome (18,24-27).

The USMF surgical technique provides wide exposure of the surgical field in patients who require difficult thyroid surgery. The technique facilitates gland exposure and vessel ligation and facilitates preservation of the EBSLN, the RLN, the cricothyroid muscles, and the PG in thyroid surgery. This analysis of patients treated by USMF technique showed good preservation of the EBSLN, RLN and cricothyroid muscles, and no patients had postoperative hematoma or permanent hypoparathyroidism.

Some limitations of this study are noted: (I) this was not a randomized study; (II) the case number was small; and (III) long-term subjective and objective analyses of voice/ swallowing performance were not performed. Further studies are needed to investigate long-term changes in voice and swallowing function after strap muscle transection.

\section{Conclusions}

By providing excellent exposure of the surgical field, USMF facilitates gland exposure, vessel ligation, preservation of 
the EBSLN, RLN, cricothyroid muscles, and preservation of the PG during difficult thyroid surgery. None of the patients analyzed in this study had postoperative wound infection, flap necrosis or hematoma. The results for objective voice analysis and subjective assessments of voice and swallowing before and 2 months after surgery showed comparable outcomes in the USMF group and the midline group. Therefore, USMF can be a safe and selectable approach for difficult thyroid surgeries.

\section{Acknowledgments}

Funding: This report was supported by grants from the Kaohsiung Medical University Hospital (KMUH 1077R50, KMUH 108-8M48, KMUH SA10807c), Taiwan.

\section{Footnote}

Conflicts of Interest: All authors have completed the ICMJE uniform disclosure form (available at http://dx.doi. org/10.21037/gs.2020.02.20). Dr. Wu reports grants from Kaohsiung Medical University Hospital, Taiwan, during the conduct of the study. Dr. Huang reports grants from Kaohsiung Medical University Hospital, Taiwan, during the conduct of the study. The other authors have no conflicts of interest to declare.

Ethical Statement: The authors are accountable for all aspects of the work in ensuring that questions related to the accuracy or integrity of any part of the work are appropriately investigated and resolved. This study was approved by the Kaohsiung Medical University Hospital Institutional Review Board [No. KMUHIRB-E(I)-20190201]. All patients have provided written informed consent for publication of this manuscript and any accompanying images.

Open Access Statement: This is an Open Access article distributed in accordance with the Creative Commons Attribution-NonCommercial-NoDerivs 4.0 International License (CC BY-NC-ND 4.0), which permits the noncommercial replication and distribution of the article with the strict proviso that no changes or edits are made and the original work is properly cited (including links to both the formal publication through the relevant DOI and the license). See: https://creativecommons.org/licenses/by-nc-nd/4.0/.

\section{References}

1. Pelizzo MR, Variolo M, Bernardi C, et al. Complications in thyroid resurgery: a single institutional experience on 233 patients from a whole series of 4,752 homogeneously treated patients. Endocrine 2014;47:100-6.

2. Campbell MJ, McCoy KL, Shen WT, et al. A multiinstitutional international study of risk factors for hematoma after thyroidectomy. Surgery 2013;154:128389; discussion 1289-91.

3. Sugino K, Nagahama M, Kitagawa W, et al. Change of surgical strategy for Graves' disease from subtotal thyroidectomy to total thyroidectomy: a single institutional experience. Endocr J 2019;66:181-6.

4. Liu J, Sun W, Dong W, et al. Risk factors for postthyroidectomy haemorrhage: a meta-analysis. Eur J Endocrinol 2017;176:591-602.

5. Deshmukh A, Gangiti K, Pantvaidya G, et al. Surgical outcomes of thyroid cancer patients in a tertiary cancer center in India. Indian J Cancer 2018;55:23-32.

6. Goncalves Filho J, Zafereo ME, Ahmad FI, et al. Decision making for the central compartment in differentiated thyroid cancer. Eur J Surg Oncol 2018;44:1671-8.

7. Fogarty BJ, Khan K, Ashall G, et al. Complications of long operations: a prospective study of morbidity associated with prolonged operative time (> 6 h). Br J Plast Surg 1999;52:33-6.

8. Vilkman E, Sonninen A, Hurme P, et al. External laryngeal frame function in voice production revisited: a review. J Voice 1996;10:78-92.

9. Sonninen AA. The role of the external laryngeal muscles in length-adjustment of the vocal cords in singing; phoniatric, roentgenologic and experimental studies of the mechanism of pitch change in the voice with special reference to the function of the sternothyroid. Acta Otolaryngol Suppl 1956;130:1-102.

10. Jaffe V, Young AE. Strap muscles in thyroid surgery: to cut or not to cut? Ann R Coll Surg Engl 1993;75:118.

11. McIvor NP, Flint DJ, Gillibrand J, et al. Thyroid surgery and voice-related outcomes. Aust N ZJ Surg 2000;70:179-83.

12. Henry LR, Solomon NP, Howard R, et al. The functional impact on voice of sternothyroid muscle division during thyroidectomy. Ann Surg Oncol 2008;15:2027-33.

13. Lee HS, Kim SW, Park HS, et al. Partial cutting of sternothyroid muscle during total thyroidectomy: impact on postoperative vocal outcomes. ScientificWorldJournal 
2013;2013:416535.

14. Banks CA, Ayers CM, Hornig JD, et al. Thyroid disease and compressive symptoms. Laryngoscope 2012;122:13-6.

15. Burns P, Timon C. Thyroid pathology and the globus symptom: are they related? A two year prospective trial. J Laryngol Otol 2007;121:242-5.

16. Borel F, Christou N, Marret O, et al. Long-term voice quality outcomes after total thyroidectomy: a prospective multicenter study. Surgery 2018;163:796-800.

17. Holler T, Anderson J. Prevalence of voice \& swallowing complaints in Pre-operative thyroidectomy patients: a prospective cohort study. J Otolaryngol Head Neck Surg 2014;43:28.

18. Stojadinovic A, Shaha AR, Orlikoff RF, et al. Prospective functional voice assessment in patients undergoing thyroid surgery. Ann Surg 2002;236:823-32.

19. Soylu L, Ozbas S, Uslu HY, et al . The evaluation of the causes of subjective voice disturbances after thyroid surgery. Am J Surg 2007;194:317-22.

20. Borel F, Tresallet C, Hamy A, et al. Self-assessment of voice outcomes after total thyroidectomy using the Voice Handicap Index questionnaire: Results of a prospective multicenter study. Surgery 2020;167:129-36.

Cite this article as: Ko HY, Lu IC, Chang PY, Wang LF, Wu CW, Yu WH, Hwang TZ, Wang CC, Huang TY, Chiang FY. U-shaped strap muscle flap for difficult thyroid surgery. Gland Surg 2020;9(2):372-379. doi: 10.21037/gs.2020.02.20
21. Lee JC, Breen D, Scott A, et al. Quantitative study of voice dysfunction after thyroidectomy. Surgery 2016;160:1576-81.

22. Lang BH, Wong CK, Ma EP. A systematic review and meta-analysis on acoustic voice parameters after uncomplicated thyroidectomy. Laryngoscope 2016;126:528-37.

23. Lombardi CP, Raffaelli M, D'Alatri L, et al. Voice and swallowing changes after thyroidectomy in patients without inferior laryngeal nerve injuries. Surgery 2006;140:1026-32; discussion 1032-4.

24. Debruyne F, Ostyn F, Delaere P, et al. Acoustic analysis of the speaking voice after thyroidectomy. J Voice 1997;11:479-82.

25. Hong KH, Kim YK. Phonatory characteristics of patients undergoing thyroidectomy without laryngeal nerve injury. Otolaryngol Head Neck Surg 1997;117:399-404.

26. Sinagra DL, Montesinos MR, Tacchi VA, et al. Voice changes after thyroidectomy without recurrent laryngeal nerve injury. J Am Coll Surg 2004;199:556-60.

27. Hodin R, Clark O, Doherty G, et al. Voice issues and laryngoscopy in thyroid surgery patients. Surgery 2013;154:46-7. 